postictal drowsiness; and 3) neurological deficits, the most reliable indicator. The mean age was 12.2 months and 12.9 months in patients with and without meningitis, respectively. CSF findings with meningitis were as follows: mean cell count $73.8 \mathrm{~mm} 3$; protein $92.6 \mathrm{~g} / \mathrm{dl}$; glucose $51.2 \mathrm{~g} / \mathrm{dl}$; all cultures were sterile, probably due to prior antibiotics. Blood cultures showed no bacteremia. In 2 patients with meningitis, cause of fever was not identified. Most common causes of fever in the total cohort were URI infection, gastritis, pneumonia, and otitis media. (Batra P, Gupta S, Gomber S, Saha A. Predictors of meningitis in children presenting with first febrile seizures. Pediatr Neurol January 2011;44:35-39). (Respond: Dr Batra, Gaur Green City, C-1019, Indirapuram, Ghaziabad, Uttar Pradesh, India. E-mail: drprernabatra@yahoo.com).

COMMENT. The authors conclude that lumbar puncture may be indicated in children aged 6-18 months who present with a first seizure with fever, especially if the seizure lasts $>30$ minutes, there is postictal drowsiness. or most specifically, there are neurological deficits. The diagnosis of meningitis was made without identifying an organism in 2 patients, and the organism identified was not specified in the remaining 3 cases. Although the CSF gram stain and culture were considered the gold standard for a diagnosis of meningitis, the diagnosis was based mainly on the CSF cell count, protein, and sugar.

Normative CSF profiles of febrile infants, aged 1 to 90 days, with negative bacterial culture and negative PCR for enteroviral infection, were studied at University of Utah, Salt Lake City. (Byington CL et al. J Pediatr Jan 2011;158:33-37). In 677 infants with atraumatic LP, the mean and median CSF WBC were $4.3 / \mathrm{mm} 3$ and $3.0 / \mathrm{mm} 3$, respectively, with a range from 0 to $12 / \mathrm{mm} 3$. CSF WBC counts were higher in the first month vs months 2 and $3 ; 6.1$ v 3.1 and 3.0, respectively. Protein levels were $75.4 \mathrm{mg} / \mathrm{dl}$ vs 58.9 and 39.2, respectively. $(\mathrm{P}<0.001$ for all). CSF glucose levels were lower in the first month compared to month $3 ; 45.3 \mathrm{mg} / \mathrm{dl}$ vs $57.7 \mathrm{mg} / \mathrm{dl}(\mathrm{P}<0.001)$. Normal CSF WBC counts in febrile infants 1 to 90 days are lower than reported in pediatric references.

\title{
FEVER-INDUCED EPILEPTIC ENCEPHALOPATHY (FIRES)
}

Clinical and imaging characteristics of fever-induced refractory epileptic encephalopathy (FIRES), idiopathic hemiconvulsion-hemiplegia syndrome (IHHS), and related acute encephalopathies are reviewed by researchers at Descartes University, Paris, France; Institute for Pharmacological Research, Milan, Italy: and Institute of Biomedical Imaging, Orsay, France. FIRES begins in school-age children with status epilepticus lasting several weeks, involving perisylvian and mesial temporal structures, and is followed by refractory epilepsy and major cognitive deterioration. IHHS begins in infancy with unilateral clonic status epilepticus and is followed by hemiplegia with refractory epilepsy. The etiology is unknown, but both FIRES and IHHS are acute encephalopathies with inflammation-mediated status epilepticus. The ketogenic diet is recommended to treat both seizures and inflammation. (Nabbout R, Vezzani A, Dulac O, Chiron C. Acute encephalopathy with inflammation-mediated status epilepticus. Lancet Neurol Jan 2011;10:99-108).Respond: Dr R Nabbout. E-mail: rimanabbout@yahoo.com 